\title{
Method for Pythagorean Interval 2-Tuple Linguistic Multiattribute Group Decision Making and Its Application to the Ship Navigation Environment Safety Assessment
}

\author{
Fuchun Yuan \\ Faculty of Transportation Engineering, Wuhan Institute of Shipbuilding Technology, Wuhan 430050, Hubei, China \\ Correspondence should be addressed to Fuchun Yuan; yuanfuchun2020@126.com
}

Received 29 November 2021; Accepted 20 January 2022; Published 25 February 2022

Academic Editor: Juan L. G. Guirao

Copyright (c) 2022 Fuchun Yuan. This is an open access article distributed under the Creative Commons Attribution License, which permits unrestricted use, distribution, and reproduction in any medium, provided the original work is properly cited.

\begin{abstract}
In this paper, some fundamental theories of the Pythagorean interval 2-tuple linguistic numbers (PI2TLNs) combined with the EDAS (evaluation based on distance from average solution) method are proposed. Firstly, we introduced the original concepts of Pythagorean interval 2-tuple linguistic sets (PI2TLSs), and two aggregation operators of PI2TLN were also introduced. Then, the steps of the EDAS method were described briefly. Moreover, the PI2TLN-EDAS model was provided to address the multiattribute group decision-making (MAGDM) problem, and calculation steps were deduced in detail. The effectiveness of the method was illustrated by taking the ship navigation environment safety assessment as an example. Finally, we conducted comparative analysis between PI2TLN-EDAS method and Pythagorean interval 2-tuple linguistic weighted averaging (PI2TLWA) operator/Pythagorean interval 2-tuple linguistic weighted geometric (PI2TLWG) operator.
\end{abstract}

\section{Introduction}

The EDAS method proposed by Ghorabaee et al. [1] is an evaluation method that calculates the positive and negative distances between each alternative and the average alternative. Different methods are used to deal with the multiattribute decision-making (MADM) problems where the decision information is a certain number [2-7]. Similar to the VIKOR method [8-12], TOPSIS method [13-17], and GRA method [18], the EDAS method is effective when conflicting criteria are present in the MADM issues. Aiming at the case where the decision information is a definite number [19-21], the EDAS method is proposed. Based on this idea, many scholars extended the EDAS method to the fuzzy set environment. For example, Kahraman et al. [22] extended the classical EDAS method to ordinary fuzzy sets. Liang et al. [23] used the EDAS-ELECTRE comprehensive evaluation method to evaluate the clean production performance of the gold mining enterprises. Jiang et al. [24] built the EDAS method based on cumulative prospect theory for MAGDM under picture fuzzy environment. Peng et al.
[25] applied the EDAS method to solve the interval value fuzzy soft decision problem. Huang et al. [26] defined an enhancement EDAS method based on prospect theory. Wei et al. [27] built the EDAS method for probabilistic linguistic MAGDM. Lei et al. [28] proposed the PDHL-EDAS method for MAGDM.

In the process of decision making, there is a wide range of ambiguity and uncertainty [29-33] such as classical fuzzy set [34], intuitionistic fuzzy sets (IFSs) [35-41], probabilistic hesitant fuzzy set [42-47], probabilistic linguistic sets [48-53], and probabilistic double hierarchy linguistic sets [54-56]. Yager [57] put forward the concept of Pythagorean fuzzy set (PFSs). Zhang [58] defined the accuracy function and new aggregation operator of Pythagorean fuzzy numbers (PFNs) and proposed the similarity measurement method of PFNs based on the method of similarity measure. Zeng et al. [59] combined the distance measure with the PFS and proposed the Pythagorean ordered weighted, average weighted, and average distance operators. Garg [60] introduced the statistical concept of confidence level into the Pythagorean fuzzy set. To better provide decision 
information in a form that is consistent with the language habits of decision makers, Huang and Wei [61] defined the Pythagorean 2-tuple linguistic fuzzy sets (P2TLSs) and introduced its related properties. The concept of Pythagorean interval 2-tuple linguistic sets (PI2TLSs) first appeared [61] on the basis of PFSs [62] and 2-tuple linguistic information [63].

Because the EDAS method is an effective MADM method and Pythagorean interval 2-tuple linguistic fuzzy set has the characteristics of two-stage description, it can relatively contain more decision-making information and describe objective facts more comprehensively and accurately. Based on the advantages of both, this paper attempts to extend the EDAS method to decision environment of Pythagorean interval 2-tuple linguistic fuzzy set (PI2TLS) and proposes a Pythagorean interval 2-tuple linguistic MADM model based on the EDAS method.

The rest of this paper is arranged as follows. In Section 2, some fundamental definitions of Pythagorean interval 2tuple linguistic numbers (PI2TLNs) are given. In Section 3, the EDAS method is extended using PI2TLNs. In Section 4, a case study for evaluating the ship navigation environment safety is given. Section 5 gives some conclusions.

\section{Preliminaries}

2.1. Pythagorean Interval 2-Tuple Linguistic Sets. The concept of Pythagorean interval 2-tuple linguistic sets (PI2TLSs) first appeared [61] on the basis of PFSs [62] and 2-tuple linguistic information [63].

Definition 1 (see [61]). In a PI2TLS, $A$ in the theoretical field $X$ is defined as follows:

$$
A=\left\{\left[\left(\psi_{(x)}, \alpha_{(x)}\right),\left(\delta_{(x)}, \beta_{(x)}\right)\right],\left(u_{A}(x), v_{A}(x)\right), x \in X\right\},
$$

where $\left[\left(\psi_{(x)}, \alpha_{(x)}\right),\left(\delta_{(x)}, \beta_{(x)}\right)\right]$ is an interval 2-tuple linguistic variable, $\psi_{(x)}, \delta_{(x)} \in S=\left\{S_{0}, S_{1} \cdots S_{T}\right\}, S$ is a linguistic term set, and $\alpha_{(x)}, \beta_{(x)} \in[-0.5,0.5), u_{A}(x), v_{A}(x) \in[0,1]$ represent the degree of membership and degree of nonmembership of the element $x$ to an interval 2-tuple linguistic $\left[\left(\psi_{(x)}, \alpha_{(x)}\right),\left(\delta_{(x)}, \beta_{(x)}\right)\right]$ and meet the condition $0 \leq u_{A}$ $(x)^{2}+v_{A}(x)^{2} \leq 1 \in[0,1]$.
For convenience, $A=\left\langle\left[\left(\psi_{A}, \alpha_{A}\right),\left(\delta_{A}, \beta_{A}\right)\right],\left(u_{A}, v_{A}\right)\right\rangle$ can be called a Pythagorean interval 2-tuple linguistic number (PI2TLN).

Definition 2 (see [61]). For a PI2TLN $\tilde{\mathrm{a}}=\left\langle\left[\left(\psi_{a}, \alpha_{a}\right),\left(\delta_{a}\right.\right.\right.$, $\left.\left.\left.\beta_{a}\right)\right],\left(u_{a}, v_{a}\right)\right\rangle$, its score function is

$$
S f(\widetilde{a})=\Delta\left(\frac{\Delta^{-1}\left(\psi_{a}, \alpha_{a}\right)+\Delta^{-1}\left(\delta_{a}, \beta_{a}\right)}{2} \times \frac{1+\left(u_{a}\right)^{2}-\left(\nu_{a}\right)^{2}}{2}\right) .
$$

Definition 3 (see [61]). For a PI2TLN $\tilde{\mathrm{a}}=\left\langle\left[\left(\psi_{a}, \alpha_{a}\right),\left(\delta_{a}\right.\right.\right.$, $\left.\left.\left.\beta_{a}\right)\right],\left(u_{a}, v_{a}\right)\right\rangle$, its accuracy function is

$$
\operatorname{Af}(\widetilde{a})=\Delta\left(\frac{\Delta^{-1}\left(\psi_{a}, \alpha_{a}\right)+\Delta^{-1}\left(\delta_{a}, \beta_{a}\right)}{2} \times \frac{\left(u_{a}\right)^{2}+\left(\nu_{a}\right)^{2}}{2}\right) \text {. }
$$

Definition 4 (see [61]). For two PI2TLNs $\tilde{a}_{1}=\left\langle\left[\left(\psi_{a_{1}}, \alpha_{a_{1}}\right)\right.\right.$, $\left.\left.\left(\delta_{a_{1}}, \beta_{a_{1}}\right)\right],\left(u_{a_{1}}, v_{a_{1}}\right)\right\rangle$ and $\tilde{a}_{2}=\left\langle\left[\left(\psi_{a_{2}}, \alpha_{a_{2}}\right),\left(\delta_{a_{2}}, \beta_{a_{2}}\right)\right],\left(u_{a_{2}}\right.\right.$, $\left.\left.v_{a_{2}}\right)\right\rangle$, respectively, the scores of $\widetilde{a}_{1}$ and $\tilde{a}_{2}$ are $S f\left(\tilde{a}_{1}\right)=$ $\Delta\left(\Delta^{-1}\left(\psi_{a_{1}}, \alpha_{a_{1}}\right)+\Delta^{-1}\left(\delta_{a_{1}}, \beta_{a_{1}}\right) / 2 \times 1+\left(u_{a_{1}}\right)^{2}-\left(\nu_{a_{1}}\right)^{2} / 2\right)$ and $S f\left(\tilde{a}_{2}\right)=\Delta\left(\Delta^{-1}\left(\psi_{a_{2}}, \alpha_{a_{2}}\right)+\Delta^{-1}\left(\delta_{a_{2}}, \beta_{a_{2}}\right) / 2 \times 1+\left(u_{a_{2}}\right)^{2}-\left(v_{a_{2}}\right)^{2}\right.$ /2), and let Af $\left(\tilde{a}_{1}\right)=\Delta\left(\Delta^{-1^{2}}\left(\psi_{a_{1}}, \alpha_{a_{1}}\right)+\Delta^{-1}\left(\delta_{a_{1}}, \beta_{a_{1}}\right) / 2 \times\right.$ $\left.\left(u_{a_{1}}\right)^{2}+\left(\nu_{a_{1}}\right)^{2} / 2\right)$ and $A f\left(\widetilde{a}_{2}\right)=\Delta\left(\Delta^{-1}\left(\psi_{a_{1}}, \alpha_{a_{1}}\right)+\Delta^{-1}\left(\delta_{a_{2}}\right.\right.$, $\left.\left.\beta_{a_{2}}\right)_{1} / 2 \times\left(u_{a_{2}}\right)^{2}+\left(\nu_{a_{2}}\right)^{2} / 2\right)$ be the accuracy degrees of $\tilde{a}_{1}$ and $\tilde{a}_{2}$; then, some operational laws of PI2TLNs are defined as follows:

(1) If $S f\left(\widetilde{a}_{1}\right)<S f\left(\widetilde{a}_{2}\right), \widetilde{a}_{1}<\widetilde{a}_{2}$.

(2) If $S f\left(\tilde{a}_{1}\right)>S f\left(\tilde{a}_{2}\right), \tilde{a}_{1}>\tilde{a}_{2}$.

(3) If $S f\left(\tilde{a}_{1}\right)=S f\left(\tilde{a}_{2}\right), A f\left(\tilde{a}_{1}\right)<A f\left(\tilde{a}_{2}\right)$, then $\tilde{a}_{1}<\tilde{a}_{2}$.

(4) If $S f\left(\tilde{a}_{1}\right)=S f\left(\tilde{a}_{2}\right), A f\left(\tilde{a}_{1}\right)>A f\left(\tilde{a}_{2}\right)$, then $\tilde{a}_{1}>\tilde{a}_{2}$.

(5) If $S f\left(\tilde{a}_{1}\right)=S f\left(\tilde{a}_{2}\right), A f\left(\tilde{a}_{1}\right)=A f\left(\tilde{a}_{2}\right)$, then $\tilde{a}_{1}=\tilde{a}_{2}$.

Definition 5 (see [61]). For two PI2TLNs $\tilde{a}_{1}=\left\langle\left[\left(\psi_{a_{1}}, \alpha_{a_{1}}\right)\right.\right.$, $\left.\left.\left(\delta_{a_{1}}, \beta_{a_{1}}\right)\right],\left(u_{a_{1}}, v_{a_{1}}\right)\right\rangle$ and $\tilde{a}_{2}=\left\langle\left[\left(\psi_{a_{2}}, \alpha_{a_{2}}\right),\left(\delta_{a_{2}}, \beta_{a_{2}}\right)\right],\left(u_{a_{2}}\right.\right.$, $\left.\left.v_{a_{2}}\right)\right\rangle$, the normalized Hamming distance $\left(H_{d}\right)$ between $\widetilde{a}_{1}$ and $\tilde{a}_{2}$ is defined as

$$
\left.H_{d}\left(\widetilde{a}_{1}, \widetilde{a}_{2}\right)=\frac{1}{4 L}\left[\mid \begin{array}{l}
\left(1+\left(u_{a_{1}}\right)^{2}-\left(v_{a_{1}}\right)^{2}\right) \cdot\left(\Delta^{-1}\left(\psi_{a_{1}}, \alpha_{a_{1}}\right)+\Delta^{-1}\left(\delta_{a_{1}}, \beta_{a_{1}}\right)\right)-\mid \\
\left(1+\left(u_{a_{2}}\right)^{2}-\left(v_{a_{2}}\right)^{2}\right) \cdot\left(\Delta^{-1}\left(\psi_{a_{2}}, \alpha_{a_{2}}\right)+\Delta^{-1}\left(\delta_{a_{2}}, \beta_{a_{2}}\right)\right)
\end{array}\right]\right],
$$


where $L$ represents the length of the language scale. It is a numerical value.
Definition 6 (see [61]). For two PI2TLNs $\widetilde{a}_{1}=\left\langle\left[\left(\psi_{a_{1}}, \alpha_{a_{1}}\right)\right.\right.$, $\left.\left.\left(\delta_{a_{1}}, \beta_{a_{1}}\right)\right],\left(u_{a_{1}}, v_{a_{1}}\right)\right\rangle$ and $\tilde{a}_{2}=\left\langle\left[\left(\psi_{a_{2}}, \alpha_{a_{2}}\right),\left(\delta_{a_{2}}, \beta_{a_{2}}\right)\right],\left(u_{a_{2}}\right.\right.$, $\left.\left.v_{a_{2}}\right)\right\rangle$, then

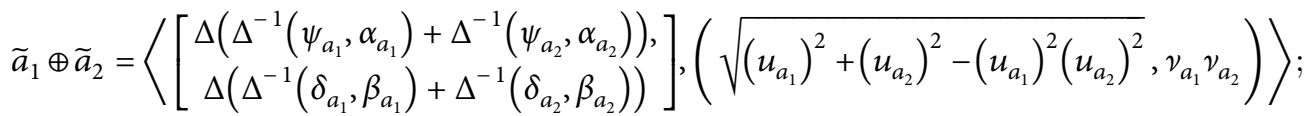

$$
\begin{aligned}
& \tilde{a}_{1} \otimes \tilde{a}_{2}=\left\langle\left[\begin{array}{c}
\Delta\left(\Delta^{-1}\left(\psi_{a_{1}}, \alpha_{a_{1}}\right) \times \Delta^{-1}\left(\psi_{a_{2}}, \alpha_{a_{2}}\right)\right), \\
\Delta\left(\Delta^{-1}\left(\delta_{a_{1}}, \beta_{a_{1}}\right) \times \Delta^{-1}\left(\delta_{a_{2}}, \beta_{a_{2}}\right)\right)
\end{array}\right],\left(u_{a_{1}} u_{a_{2}}, \sqrt{\left(v_{a_{1}}\right)^{2}+\left(v_{a_{2}}\right)^{2}-\left(v_{a_{1}}\right)^{2}\left(v_{a_{2}}\right)^{2}}\right)\right\rangle ; \\
& \lambda \widetilde{a}_{1}=\left\langle\left[\begin{array}{c}
\Delta\left(\lambda \Delta^{-1}\left(\psi_{a_{1}}, \alpha_{a_{1}}\right)\right), \\
\Delta\left(\lambda \Delta^{-1}\left(\delta_{a_{1}}, \beta_{a_{1}}\right)\right)
\end{array}\right],\left(\sqrt{1-\left(1-\left(u_{a_{1}}\right)^{2}\right)^{\lambda}},\left(v_{a_{1}}\right)^{\lambda}\right)\right\rangle ; \\
& \left(\widetilde{a}_{1}\right)^{\lambda}=\left\langle\left[\begin{array}{c}
\Delta\left(\left(\Delta^{-1}\left(\psi_{a_{1}}, \alpha_{a_{1}}\right)\right)^{\lambda}\right), \\
\Delta\left(\left(\Delta^{-1}\left(\delta_{a_{1}}, \beta_{a_{1}}\right)\right)^{\lambda}\right)
\end{array}\right],\left(\left(u_{a_{1}}\right)^{\lambda}, \sqrt{1-\left(1-\left(v_{a_{1}}\right)^{2}\right)^{\lambda}}\right)\right\rangle \text {. }
\end{aligned}
$$

2.2. Pythagorean Interval 2-Tuple Linguistic Arithmetic Aggregation Operators. In this section, the PI2TLWA operator and PI2TLWG operator are introduced.
Definition 7 (see [64]). For $\tilde{a}_{j}=\left\langle\left[\left(\psi_{a_{i}}, \alpha_{a_{j}}\right),\left(\delta_{a_{j}}\right.\right.\right.$, $\left.\left.\left.\beta_{a_{i}}\right)\right],\left(u_{a_{i}}, v_{a_{j}}\right)\right\rangle(j=1,2, \ldots, n)$ is a collection of PI2TLNs. PI2TLWA operator is defined as

$$
\begin{aligned}
\operatorname{PI} 2 \operatorname{TLWA}_{\omega}\left(\tilde{a}_{1}, \tilde{a}_{2}, \ldots, \tilde{a}_{n}\right) & =\underset{j=1}{\oplus}\left(\omega_{j} \tilde{a}_{j}\right) \\
& =\left\langle\left[\begin{array}{c}
\Delta\left(\sum_{j=1}^{n} \omega_{j} \Delta^{-1}\left(\psi_{a_{j}}, \alpha_{a_{j}}\right)\right) \\
\Delta\left(\sum_{j=1}^{n} \omega_{j} \Delta^{-1}\left(\delta_{a_{j}}, \beta_{a_{j}}\right)\right)
\end{array}\right],\left(\sqrt{\left.\left.1-\prod_{j=1}^{n}\left(1-\left(u_{a_{j}}\right)^{2}\right)^{\omega_{j}}, \prod_{j=1}^{n}\left(v_{a_{j}}\right)^{\omega_{j}}\right)\right\rangle,}\right.\right.
\end{aligned}
$$

where $\omega=\left(\omega_{1}, \omega_{2}, \ldots, \omega_{n}\right)^{T}$ is the weight vector of $\widetilde{a}_{j}(j=$ $1,2, \ldots, n)$ and $\omega_{j}>0, \sum_{j=1}^{n} \omega_{j}=1$.

$$
\begin{aligned}
\text { PI } 2 \operatorname{TLWG}_{\omega}\left(\widetilde{a}_{1}, \tilde{a}_{2}, \ldots, \tilde{a}_{n}\right) & =\underset{j=1}{\otimes}\left(\omega_{j} \tilde{a}_{j}\right) \\
& =\left\langle\begin{array}{c}
\Delta\left(\prod_{j=1}^{n} \Delta^{-1}\left(\psi_{a_{j}}, \alpha_{a_{j}}\right)^{\omega_{j}}\right) \\
\Delta\left(\prod_{j=1}^{n} \Delta^{-1}\left(\delta_{a_{j}}, \beta_{a_{j}}\right)^{\omega_{j}}\right)
\end{array}\right],\left(, \prod_{j=1}^{n}\left(u_{a_{j}}\right)^{\omega_{j}} \sqrt{\left.\left.1-\prod_{j=1}^{n}\left(1-\left(v_{a_{j}}\right)^{2}\right)^{\omega_{j}}\right)\right\rangle,}\right.
\end{aligned}
$$

Definition 8 (see [31]). For $\tilde{a}_{j}=\left\langle\left[\left(\psi_{a_{j}}, \alpha_{a_{j}}\right),\left(\delta_{a_{i}}, \beta_{a_{j}}\right)\right]\right.$, $\left.\left(u_{a_{i}}, v_{a_{i}}\right)\right\rangle(j=1,2, \ldots, n)$ is a collection of PI2TLNs. PI2TLWG operator is defined as where $\omega=\left(\omega_{1}, \omega_{2}, \ldots, \omega_{n}\right)^{T}$ is the weight vector of $\widetilde{a}_{j}(j=$ $1,2, \ldots, n)$ and $\omega_{j}>0, \sum_{j=1}^{n} \omega_{j}=1$.

\section{EDAS Method for MAGDM with PI2TLNs}

Let $P=\left\{P_{1}, P_{2}, \ldots, P_{m}\right\}$ be a solution set, $\sigma=\left\{\sigma_{1}, \sigma_{2}, \ldots\right.$, $\left.\sigma_{n}\right\}$ be an evaluation attribute set, and $D=\left\{D_{1}, D_{2}, \ldots, D_{k}\right\}$ be an expert set. $\left\{\varpi_{1}, \varpi_{2}, \ldots, \varpi_{k}\right\}$ and $\left\{\omega_{1}, \omega_{2}, \ldots, \omega_{n}\right\}$ are, 


$$
T^{(k)}=\left[t_{i j}^{k}\right]_{m \times n}=\left[\begin{array}{cccc}
t_{11}^{k} & t_{12}^{k} & \ldots & t_{1 n}^{k} \\
t_{21}^{k} & t_{22}^{k} & \ldots & t_{2 n}^{k} \\
\vdots & \vdots & \vdots & \vdots \\
t_{m 1}^{k} & t_{m 2}^{k} & \ldots & t_{m n}^{k}
\end{array}\right] .
$$

Step 2. Use PI2TLWA operator or PI2TLWG operator to obtain the group evaluation matrix $T=\left(t_{i j}\right)_{m \times n}$ :

$$
\begin{aligned}
T & =[t E]_{m \times n}=\left[\begin{array}{cccc}
t_{11} & t_{12} & \ldots & t_{1 n} \\
t_{21} & t_{22} & \ldots & t_{2 n} \\
\vdots & \vdots & \vdots & \vdots \\
t_{m 1} & t_{m 2} & \ldots & t_{\mathrm{mn}}
\end{array}\right], \\
t_{i j} & =\underset{t=1}{\oplus} t_{i j}^{k}=P I 2 \operatorname{TLWA}\left(t_{i j}^{1}, t_{i j}^{2}, \ldots, t_{i j}^{k}\right) \\
& =\left\langle\begin{array}{r}
\Delta\left(\sum_{t=1}^{k} \Phi_{t} \Delta^{-1}\left(\psi_{g_{i j}}^{(t)}, \alpha_{g_{i j}}^{(t)}\right)\right), \\
\Delta\left(\sum_{t=1}^{k} \varpi_{t} \Delta^{-1}\left(\delta_{g_{i j}}^{(t)}, \beta_{g_{i j}}^{(t)}\right)\right)
\end{array}\right],\left(\sqrt{\left.\left.1-\prod_{t=1}^{k}\left(1-\left(u_{g_{i j}}^{(t)}\right)^{2}\right)^{\omega_{t}}, \prod_{t=1}^{k}\left(v_{g_{i j}}^{(t)}\right)^{\omega_{t}}\right)\right\rangle,}\right.
\end{aligned}
$$

or

$$
\begin{aligned}
t_{i j} & =\otimes_{t=1}^{\otimes} t_{i j}^{k}=\operatorname{P} 2 \operatorname{TLWG}\left(t_{i j}^{1}, t_{i j}^{2}, \ldots, t_{i j}^{k}\right) \\
& =\left\langle\left[\begin{array}{c}
\Delta\left(\sum_{t=1}^{k} \Delta^{-1}\left(\psi_{g_{i j}}^{(t)}, \alpha_{g_{i j}}^{(t)}\right)^{\Phi_{t}}\right) \\
\Delta\left(\sum_{t=1}^{k} \Delta^{-1}\left(\delta_{g_{i j}}^{(t)}, \beta_{g_{i j}}^{(t)}\right)^{\omega_{t}}\right)
\end{array}\right],\left(, \prod_{t=1}^{k}\left(u_{g_{i j}}^{(t)}\right)^{\omega_{t}} \sqrt{\left.\left.1-\prod_{t=1}^{k}\left(1-\left(v_{g_{i j}}^{(t)}\right)^{2}\right)^{\omega_{t}}\right)\right\rangle .}\right.\right.
\end{aligned}
$$

Step 3. Calculate $A V$ :

According to Definition 6,

$$
\mathrm{AV}=\left[\mathrm{AV}_{j}\right]_{1 \times n}=\left[\frac{\sum_{i=1}^{m} t_{i j}}{m}\right]_{1 \times n} .
$$

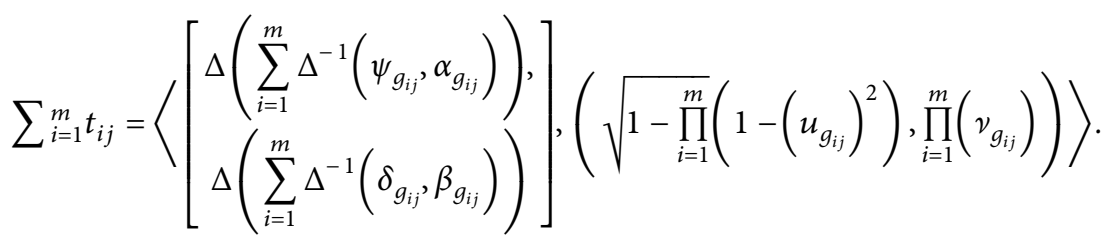

Thus, 


$$
\begin{aligned}
A V & =\left[A V_{j}\right]_{1 \times n}=\left[\frac{\sum_{i=1}^{m} t_{i j}}{m}\right]_{1 \times n} \\
& =\left\langle\begin{array}{l}
\Delta\left(\sum_{i=1}^{m} \frac{1}{m} \Delta^{-1}\left(\psi_{g_{i j}}, \alpha_{g_{i j}}\right)\right), \\
\Delta\left(\sum_{i=1}^{m} \frac{1}{m} \Delta^{-1}\left(\delta_{g_{i j}}, \beta_{g_{i j}}\right)\right)
\end{array}\right],\left(\sqrt{\left.\left.1-\prod_{i=1}^{m}\left(1-\left(u_{g_{i j}}\right)^{2}\right)^{1 / m}, \prod_{i=1}^{m}\left(v_{g_{i j}}\right)^{1 / m}\right)\right\rangle .}\right.
\end{aligned}
$$

Step 4. According to AV, calculate the values of PDA and NDA depending on the type of attribute.

$$
\begin{aligned}
& \mathrm{PDA}_{i j}=\left[\mathrm{PDA}_{i j}\right]_{m \times n}=\frac{\max \left(0,\left(t_{i j}-A V_{j}\right)\right)}{A V_{j}}, \\
& \mathrm{NDA}_{i j}=\left[\mathrm{NDA}_{i j}\right]_{m \times n}=\frac{\max \left(0,\left(A V_{j}-t_{i j}\right)\right)}{A V_{j}} .
\end{aligned}
$$

To facilitate calculation, the PI2TLNs' scoring function proposed in Definition 2 is used to calculate the PDA and NDA values as follows:

$$
\begin{aligned}
\mathrm{PDA}_{i j} & =\left[\mathrm{PDA}_{i j}\right]_{m \times n}=\frac{\max \left(0,\left(s\left(t_{i j}\right)-s\left(A V_{j}\right)\right)\right)}{s\left(A V_{j}\right)}, \\
\mathrm{NDA}_{i j} & =\left[\mathrm{NDA}_{i j}\right]_{m \times n}=\frac{\max \left(0,\left(s\left(A V_{j}\right)-s\left(t_{i j}\right)\right)\right)}{s\left(A V_{j}\right)} .
\end{aligned}
$$

Step 5. Calculate weighting distance $\mathrm{SP}_{i}$ and $\mathrm{SN}_{i}$.

$$
\begin{aligned}
& \mathrm{SP}_{i}=\sum_{j=1}^{n} \omega_{j} \mathrm{PDA}_{i j}, \\
& \mathrm{SN}_{i}=\sum_{j=1}^{n} \omega_{j} \mathrm{NDA}_{i j} .
\end{aligned}
$$

Step 6. Normalize the calculating results of the following equation:

$$
\begin{aligned}
\mathrm{NSP}_{i} & =\frac{\mathrm{SP}_{i}}{\max \left(\mathrm{SP}_{i}\right)}, \\
\mathrm{NSN}_{i} & =1-\frac{\mathrm{SN}_{i}}{\max \left(\mathrm{SN}_{i}\right)}
\end{aligned}
$$

Step 7. Calculate the values of appraisal score (AS).

$$
\mathrm{AS}_{i}=\frac{1}{2}\left(\mathrm{NSP}_{i}+\mathrm{NSN}_{i}\right)
$$

Step 8. All alternatives are ranked according to their final evaluation value.

\section{Numerical Example}

In recent years, due to the increase in ship types, larger ships, faster speeds, congested routes, and more bridges and port facilities, the navigation environment has undergone great changes and become more and more complex, but operators must operate in a certain environment. Based on the severe changes around sailing environment, the difficulty for operation becomes harder and harder for operators to finish tasks safely and efficiently under this environment. More mistakes will occur because the pressure of the operators becomes heavier and heavier. To prevent and reduce occurrence of accidents and to make safe and efficient transportation on the sea, not only the quality of operators should be improved and the performance and management of the ship should be improved but also the sailing environment should be improved. The improvement of environment is based on systematic analysis and evaluation of the environment, so we have to understand what the danger elements are in the sailing and what level the danger has reached, so as to take pointed measure to improve the environment. According to the relevant conclusions, ship operation simulation results, and domestic and foreign expert consultation, this paper sets the risk evaluation level for each evaluation target and uses the PI2TLN-EDAS method to evaluate the safety of the ship's navigation environment and then to evaluate the safety of the ship's navigation environment. Obviously, evaluation of the ship navigation environment safety is a classical MAGDM problem. In this section, a numerical example is provided to evaluate the ship navigation environment safety by using EDAS method with PI2TLNs. Assume that there are five possible ship navigations $P_{i}(i=1,2,3,4,5)$ to be assessed by four evaluation attributes $\sigma_{j}(j=1,2,3,4):(1) \sigma_{1}$ is the hydrometeorological factor; (2) $\sigma_{2}$ is the channel condition factor; (3) $\sigma_{3}$ is the channel traffic factor; and (4) $\sigma_{4}$ is the port condition factor. Five possible ship navigations $P_{i}(i=1,2,3,4,5)$ are assessed with PI2TLNs under four attributes by three experts $D^{k}$ (expert's weight $\omega=(0.25,0.39,0.36)$ and attributes' weight $\omega=(0.22,0.32,0.26,0.20))$.

In order to get the most desirable ship navigations, the relevant calculating steps are listed below: 
TABLE 1: Rating alternatives on each criterion by $D_{1}$.

\begin{tabular}{llr}
\hline & $\sigma_{1}$ & $\sigma_{2}$ \\
\hline$P_{1}$ & $<\left[\left(S_{3}, 0\right),\left(S_{4}, 0\right)\right],(0.4,0.7)>$ & $<\left[\left(S_{5}, 0\right),\left(S_{2}, 0\right)\right],(0.6,0.1)>$ \\
$P_{2}$ & $<\left[\left(S_{5}, 0\right),\left(S_{2}, 0\right)\right],(0.6,0.4)>$ & $<\left[\left(S_{3}, 0\right),\left(S_{6}, 0\right)\right],(0.6,0.5)>$ \\
$P_{3}$ & $<\left[\left(S_{5}, 0\right),\left(S_{6}, 0\right)\right],(0.2,0.6)>$ & $<\left[\left(S_{1}, 0\right),\left(S_{3}, 0\right)\right],(0.3,0.8)>$ \\
$P_{4}$ & $<\left[\left(S_{2}, 0\right),\left(S_{3}, 0\right)\right],(0.6,0.8)>$ & $<\left[\left(S_{6}, 0\right),\left(S_{2}, 0\right)\right],(0.2,0.9)>$ \\
$P_{5}$ & $<\left[\left(S_{1}, 0\right),\left(S_{1}, 0\right)\right],(0.5,0.6)>$ & $<\left[\left(S_{4}, 0\right),\left(S_{1}, 0\right)\right],(0.5,0.7)>$ \\
\hline & $\sigma_{3}$ & $\sigma_{4}$ \\
$P_{1}$ & $<\left[\left(S_{2}, 0\right),\left(S_{2}, 0\right)\right],(0.5,0.5)>$ & $<\left[\left(S_{3}, 0\right),\left(S_{1}, 0\right)\right],(0.4,0.5)>$ \\
$P_{2}$ & $<\left[\left(S_{4}, 0\right),\left(S_{5}, 0\right)\right],(0.2,0.5)>$ & $<\left[\left(S_{4}, 0\right),\left(S_{2}, 0\right)\right],(0.4,0.7)>$ \\
$P_{3}$ & $<\left[\left(S_{5}, 0\right),\left(S_{3}, 0\right)\right],(0.6,0.2)>$ & $<\left[\left(S_{3}, 0\right),\left(S_{5}, 0\right)\right],(0.2,0.4)>$ \\
$P_{4}$ & $<\left[\left(S_{1}, 0\right),\left(S_{6}, 0\right)\right],(0.3,0.3)>$ & $<\left[\left(S_{3}, 0\right),\left(S_{4}, 0\right)\right],(0.7,0.5)>$ \\
$P_{5}$ & $<\left[\left(S_{3}, 0\right),\left(S_{4}, 0\right)\right],(0.6,0.1)>$ & $<\left[\left(S_{4}, 0\right),\left(S_{2}, 0\right)\right],(0.6,0.4)>$
\end{tabular}

TABLe 2: Rating alternatives on each criterion by $D_{2}$.

\begin{tabular}{llr}
\hline & \multicolumn{1}{c}{$\sigma_{1}$} & $\sigma_{2}$ \\
\hline$P_{1}$ & $<\left[\left(S_{1}, 0\right),\left(S_{3}, 0\right)\right],(0.6,0.4)>$ & $<\left[\left(S_{1}, 0\right),\left(S_{5}, 0\right)\right],(0.5,0.7)>$ \\
$P_{2}$ & $<\left[\left(S_{3}, 0\right),\left(S_{5}, 0\right)\right],(0.3,0.6)>$ & $<\left[\left(S_{3}, 0\right),\left(S_{3}, 0\right)\right],(0.4,0.2)>$ \\
$P_{3}$ & $<\left[\left(S_{4}, 0\right),\left(S_{6}, 0\right)\right],(0.5,0.8)>$ & $<\left[\left(S_{2}, 0\right),\left(S_{6}, 0\right)\right],(0.6,0.3)>$ \\
$P_{4}$ & $<\left[\left(S_{3}, 0\right),\left(S_{2}, 0\right)\right],(0.1,0.6)>$ & $<\left[\left(S_{6}, 0\right),\left(S_{1}, 0\right)\right],(0.2,0.8)>$ \\
$P_{5}$ & $<\left[\left(S_{4}, 0\right),\left(S_{1}, 0\right)\right],(0.2,0.7)>$ & $<\left[\left(S_{4}, 0\right),\left(S_{3}, 0\right)\right],(0.3,0.9)>$ \\
\hline & $\sigma_{3}$ & $\sigma_{4}$ \\
$P_{1}$ & $<\left[\left(S_{3}, 0\right),\left(S_{1}, 0\right)\right],(0.5,0.7)>$ & $<\left[\left(S_{2}, 0\right),\left(S_{2}, 0\right)\right],(0.3,0.7)>$ \\
$P_{2}$ & $<\left[\left(S_{4}, 0\right),\left(S_{3}, 0\right)\right],(0.6,0.8)>$ & $<\left[\left(S_{4}, 0\right),\left(S_{3}, 0\right)\right],(0.6,0.2)>$ \\
$P_{3}$ & $<\left[\left(S_{1}, 0\right),\left(S_{5}, 0\right)\right],(0.7,0.5)>$ & $<\left[\left(S_{5}, 0\right),\left(S_{4}, 0\right)\right],(0.4,0.5)>$ \\
$P_{4}$ & $<\left[\left(S_{6}, 0\right),\left(S_{3}, 0\right)\right],(0.2,0.4)>$ & $<\left[\left(S_{1}, 0\right),\left(S_{5}, 0\right)\right],(0.1,0.7)>$ \\
$P_{5}$ & $<\left[\left(S_{2}, 0\right),\left(S_{4}, 0\right)\right],(0.6,0.6)>$ & $<\left[\left(S_{6}, 0\right),\left(S_{1}, 0\right)\right],(0.4,0.9)>$ \\
$P_{5}$ & $<\left[\left(S_{3}, 0\right),\left(S_{4}, 0\right)\right],(0.6,0.1)>$ & $<\left[\left(S_{4}, 0\right),\left(S_{2}, 0\right)\right],(0.6,0.4)>$ \\
\hline
\end{tabular}

TABLE 3: Rating alternatives on each criterion by $D_{3}$.

\begin{tabular}{llr}
\hline & $\sigma_{1}$ & $\sigma_{2}$ \\
\hline$P_{1}$ & $<\left[\left(S_{1}, 0\right),\left(S_{4}, 0\right)\right],(0.7,0.7)>$ & $<\left[\left(S_{5}, 0\right),\left(S_{5}, 0\right)\right],(0.7,0.3)>$ \\
$P_{2}$ & $<\left[\left(S_{6}, 0\right),\left(S_{5}, 0\right)\right],(0.8,0.4)>$ & $<\left[\left(S_{4}, 0\right),\left(S_{3}, 0\right)\right],(0.3,0.6)>$ \\
$P_{3}$ & $<\left[\left(S_{2}, 0\right),\left(S_{1}, 0\right)\right],(0.4,0.4)>$ & $<\left[\left(S_{3}, 0\right),\left(S_{5}, 0\right)\right],(0.6,0.7)>$ \\
$P_{4}$ & $<\left[\left(S_{1}, 0\right),(S 3,0)\right],(0.7,0.4)>$ & $<\left[\left(S_{5}, 0\right),\left(S_{5}, 0\right)\right],(0.4,0.4)>$ \\
$P_{5}$ & $<\left[\left(S_{5}, 0\right),\left(S_{4}, 0\right)\right],(0.5,0.4)>$ & $<\left[\left(S_{4}, 0\right),\left(S_{6}, 0\right)\right],(0.6,0.5)>$ \\
\hline & $\sigma_{3}$ & $\sigma_{4}$ \\
$P_{1}$ & $<\left[\left(S_{1}, 0\right),\left(S_{2}, 0\right)\right],(0.3,0.5)>$ & $<\left[\left(S_{3}, 0\right),\left(S_{4}, 0\right)\right],(0.3,0.3)>$ \\
$P_{2}$ & $<\left[\left(S_{4}, 0\right),\left(S_{4}, 0\right)\right],(0.5,0.8)>$ & $<\left[\left(S_{4}, 0\right),\left(S_{1}, 0\right)\right],(0.4,0.5)>$ \\
$P_{3}$ & $<\left[\left(S_{3}, 0\right),\left(S_{5}, 0\right)\right],(0.6,0.7)>$ & $<\left[\left(S_{1}, 0\right),\left(S_{2}, 0\right)\right],(0.2,0.6)>$ \\
$P_{4}$ & $<\left[\left(S_{5}, 0\right),\left(S_{5}, 0\right)\right],(0.4,0.4)>$ & $<\left[\left(S_{2}, 0\right),\left(S_{5}, 0\right)\right],(0.5,0.7)>$ \\
$P_{5}$ & $<\left[\left(S_{6}, 0\right),\left(S_{2}, 0\right)\right],(0.5,0.6)>$ & $<\left[\left(S_{2}, 0\right),\left(S_{1}, 0\right)\right],(0.6,0.6)>$ \\
\hline
\end{tabular}

TABle 4: The group Pythagorean interval 2-tuple linguistic decision matrix $R$.

\begin{tabular}{lcc}
\hline & $\sigma_{1}$ & $\sigma_{2}$ \\
\hline$P_{1}$ & $<\left[\left(S_{2},-0.5\right),\left(S_{4},-0.39\right)\right],(0.6072,0.6152)>$ & $<\left[\left(S_{3}, 0.44\right),\left(S_{4}, 0.25\right)\right],(0.6104,0.5641)>$ \\
$P_{2}$ & $<\left[\left(S_{5},-0.42\right),\left(S_{4}, 0.25\right)\right],(0.635,0.5891)>$ & $<\left[\left(S_{3}, 0.36\right),\left(S_{4},-0.25\right)\right],(0.4385,0.4442)>$ \\
$P_{3}$ & $<\left[\left(S_{4}, 0.25\right),\left(S_{6},-0.36\right)\right],(0.4965,0.7627)>$ & $<\left[\left(S_{2},-0.25\right),\left(S_{3}, 0.45\right)\right],(0.4788,0.4496)>$ \\
$P_{4}$ & $<\left[\left(S_{4},-0.17\right),\left(S_{2}, 0.25\right)\right],(0.4041,0.3577)>$ & $<\left[\left(S_{4}, 0.2\right),\left(S_{2},-0.03\right)\right],(0.4853,0.6591)>$ \\
$P_{5}$ & $<\left[\left(S_{4},-0.39\right),\left(S_{2}, 0.08\right)\right],(0.4174,0.6256)>$ & $<\left[\left(S_{4}, 0\right),\left(S_{4},-0.42\right)\right],(0.4859,0.7478)>$ \\
\hline & $\sigma_{3}$ & $\sigma_{4}$ \\
$P_{1}$ & $<\left[\left(S_{2}, 0.03\right),\left(S_{2},-0.39\right)\right],(0.4426,0.678)>$ & $<\left[\left(S_{3},-0.39\right),\left(S_{2}, 0.47\right)\right],(0.3287,0.5641)>$ \\
$P_{2}$ & $<\left[\left(S_{4}, 0\right),\left(S_{4},-0.14\right)\right],(0.5001,0.8459)>$ & $<\left[\left(S_{4}, 0\right),\left(S_{2}, 0.03\right)\right],(0.4945,0.4159)>$ \\
$P_{3}$ & $<\left[\left(S_{3},-0.28\right),\left(S_{5},-0.5\right)\right],(0.6436,0.6712)>$ & $<\left[\left(S_{3}, 0.06\right),\left(S_{4},-0.47\right)\right],(0.2979,0.6349)>$ \\
$P_{4}$ & $<\left[\left(S_{4}, 0.39\right),\left(S_{4}, 0.47\right)\right],(0.3118,0.503)>$ & $<\left[\left(S_{2},-0.14\right),\left(S_{5},-0.25\right)\right],(0.491,0.7653)>$ \\
$P_{5}$ & $<\left[\left(S_{4},-0.31\right),\left(S_{3}, 0.28\right)\right],(0.5678,0.6817)>$ & $<\left[\left(S_{4}, 0.06\right),\left(S_{1}, 0.25\right)\right],(0.537,0.7985)>$ \\
\hline
\end{tabular}


TABLE 5: The score values of $g_{i j}$ and $\mathrm{AV}_{j}$.

\begin{tabular}{lcccr}
\hline & $\sigma_{1}$ & $\sigma_{2}$ & $\sigma_{3}$ & $\sigma_{4}$ \\
\hline$P_{1}$ & 2.5300 & 4.0542 & 1.3400 & 2.0062 \\
$P_{2}$ & 4.6627 & 3.5372 & 2.1008 & 3.2306 \\
$P_{3}$ & 3.2877 & 2.6705 & 3.4792 & 2.2589 \\
$P_{4}$ & 3.1475 & 2.4714 & 3.7399 & 2.1661 \\
$P_{5}$ & 2.2270 & 2.5656 & 2.9889 & 1.7277 \\
SAV $_{j}$ & 3.3803 & 3.1769 & 2.8299 & 2.4130 \\
\hline
\end{tabular}

TABLE 6: The results of $\mathrm{PDA}_{i j}$.

\begin{tabular}{lcccc}
\hline & $\sigma_{1}$ & $\sigma_{2}$ & $\sigma_{3}$ & $\sigma_{4}$ \\
\hline$P_{1}$ & 0.0000 & 0.2761 & 0.0000 & 0.0000 \\
$P_{2}$ & 0.3794 & 0.1134 & 0.0000 & 0.3389 \\
$P_{3}$ & 0.0000 & 0.0000 & 0.2294 & 0.0000 \\
$P_{4}$ & 0.0000 & 0.0000 & 0.3216 & 0.0000 \\
$P_{5}$ & 0.0000 & 0.0000 & 0.0562 & 0.0000 \\
\hline
\end{tabular}

TABLE 7: The results of $\mathrm{NDA}_{i j}$.

\begin{tabular}{lcclc}
\hline & $\sigma_{1}$ & $\sigma_{2}$ & $\sigma_{3}$ & $\sigma_{4}$ \\
\hline$P_{1}$ & 0.2515 & 0.0000 & 0.5265 & 0.1686 \\
$P_{2}$ & 0.0000 & 0.0000 & 0.2576 & 0.0000 \\
$P_{3}$ & 0.0274 & 0.1594 & 0.0000 & 0.0638 \\
$P_{4}$ & 0.0689 & 0.2221 & 0.0000 & 0.1023 \\
$P_{5}$ & 0.3412 & 0.1924 & 0.0000 & 0.2840 \\
\hline
\end{tabular}

TABLE 8: The values of $\mathrm{SP}_{i}$ and $\mathrm{SN}_{i}$.

\begin{tabular}{lccccc}
\hline & $P_{1}$ & $P_{2}$ & $P_{3}$ & $P_{4}$ & $P_{5}$ \\
\hline $\mathrm{SP}_{i}$ & 0.0884 & 0.1875 & 0.0596 & 0.0836 & 0.0146 \\
$\mathrm{SN}_{i}$ & 0.2259 & 0.0670 & 0.0698 & 0.1067 & 0.1934 \\
\hline
\end{tabular}

TABLE 9: The values of $\mathrm{NSP}_{i}$ and $\mathrm{NSN}_{i}$.

\begin{tabular}{lccccc}
\hline & $P_{1}$ & $P_{2}$ & $P_{3}$ & $P_{4}$ & $P_{5}$ \\
\hline NSP $_{i}$ & 0.4712 & 1.0000 & 0.3181 & 0.4458 & 0.0779 \\
NSN $_{i}$ & 0.0000 & 0.7035 & 0.6911 & 0.5278 & 0.1439 \\
\hline
\end{tabular}

Step 1. Construct the evaluation matrix $T^{(k)}=\left(t_{i j}^{k}\right)_{5 \times 4}(i=1,2, \ldots, 5, j=1,2, \ldots, 4)$ of each decision maker as in Tables $1-3$.
Step 2. Use PI2TLWA operator to integrate evaluation information from decision makers (expert's weight $\Phi=$ $(0.25,0.39,0.36))$. The results are presented in Table 4 . 
Step 3. Calculate the value of $\left(\mathrm{AV}_{j}\right)$ :

$$
\left.\begin{array}{c}
\mathrm{AV}_{1}=\left\{\begin{array}{c}
{\left[\begin{array}{c}
\Delta\left(\frac{1}{5} \times 1.5000+\frac{1}{5} \times 4.5800+\frac{1}{5} \times 4.2500+\frac{1}{5} \times 3.8300+\frac{1}{5} \times 3.6100\right), \\
\Delta\left(\frac{1}{5} \times 3.6100+\frac{1}{5} \times 4.2500+\frac{1}{5} \times 5.6400+\frac{1}{5} \times 2.2500+\frac{1}{5} \times 2.0800\right)
\end{array}\right],} \\
\left(\begin{array}{c}
\sqrt{1-\left(1-0.6072^{2}\right)^{1 / 5} \times\left(1-0.6350^{2}\right)^{1 / 5} \times\left(1-0.4965^{2}\right)^{1 / 5}} \\
\rtimes\left(1-0.4041^{2}\right)^{1 / 5} \times\left(1-0.4174^{2}\right)^{1 / 5}
\end{array}\right. \\
(0.6152)^{1 / 5} \times(0.5891)^{1 / 5} \times(0.7627)^{1 / 5} \times(0.3577)^{1 / 5} \times(0.6256)^{1 / 5}
\end{array}\right)
\end{array}\right\}
$$

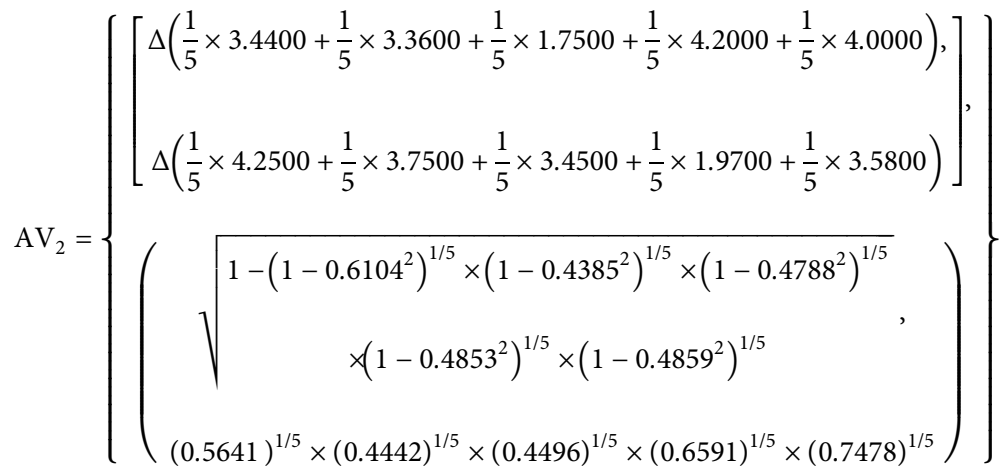

$=\left\langle\left[\left(s_{3},-0.3500\right),\left(s_{3}, 0.4000\right)\right],(0.5059,0.5609)\right\rangle$,

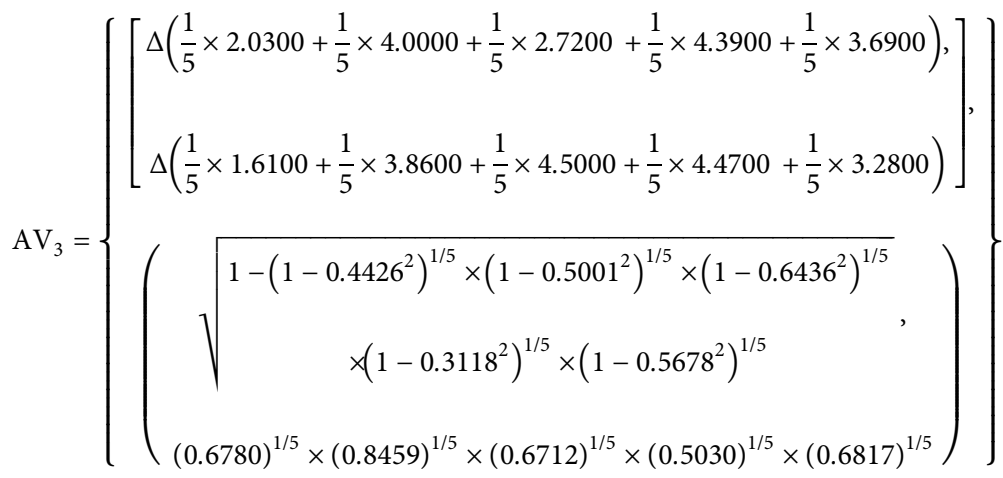

$=\left\langle\left[\left(s_{3}, 0.3660\right),\left(s_{4},-0.4560\right)\right],(0.5137,0.6670)\right\rangle$,

$$
\mathrm{AV}_{4}=\left\{\begin{array}{l}
{\left[\begin{array}{c}
\Delta\left(\frac{1}{5} \times 2.6100+\frac{1}{5} \times 4.0000+\frac{1}{5} \times 3.0600+\frac{1}{5} \times 1.8600+\frac{1}{5} \times 4.0600\right), \\
\Delta\left(\frac{1}{5} \times 2.4700+\frac{1}{5} \times 2.0300+\frac{1}{5} \times 3.5300+\frac{1}{5} \times 4.7500+\frac{1}{5} \times 1.2500\right)
\end{array}\right],} \\
\left(\begin{array}{c}
\sqrt{1-\left(1-0.3287^{2}\right)^{1 / 5} \times\left(1-0.4945^{2}\right)^{1 / 5} \times\left(1-0.2979^{2}\right)^{1 / 5}}, \\
\times\left(1-0.4910^{2}\right)^{1 / 5} \times\left(1-0.5370^{2}\right)^{1 / 5}
\end{array}\right. \\
=\left\langle\left[\left(s_{3}, 0.1180\right),\left(s_{3},-0.1940\right)\right],(0.4451,0.6192)\right\rangle .
\end{array}\right\}
$$


So, the values of $A V_{j}$ are determined as follows:

$$
\mathrm{AV}_{1 \times 4}=\left\{\begin{array}{c}
\left\langle\left[\left(s_{4},-0.4460\right),\left(s_{4},-0.4340\right)\right],(0.5273,0.5732)\right\rangle, \\
\left\langle\left[\left(s_{3},-0.3500\right),\left(s_{3}, 0.4000\right)\right],(0.5059,0.5609)\right\rangle, \\
\left\langle\left[\left(s_{3}, 0.3660\right),\left(s_{4},-0.4560\right)\right],(0.5137,0.6670)\right\rangle, \\
\left\langle\left[\left(s_{3}, 0.1180\right),\left(s_{3},-0.1940\right)\right],(0.4451,0.6192)\right\rangle
\end{array}\right\} .
$$

Step 4. Based on the results of average solution $\left(A V_{j}\right)$, equations (14) and (15) are used to calculate $\mathrm{PDA}_{\mathrm{ij}}$ and $\mathrm{NDA}_{\mathrm{ij}}$ (see Tables 5-7).

Step 5. Compute the values of $\mathrm{SP}_{i}$ and $\mathrm{SN}_{i}$ by equation (17) (weighting vector $\omega=(0.22,0.32,0.26,0.20))$, and the results are depicted in Table 8.

Step 6. Normalize the results of Step 6 by equation (18), and the results are presented in Table 9.

Step 7. Compute $\operatorname{AS}_{i}(i=1,2,3,4,5)$ :

$$
\begin{aligned}
& \mathrm{AS}_{1}=0.2356, \\
& \mathrm{AS}_{2}=0.8518, \\
& \mathrm{AS}_{3}=0.5046, \\
& \mathrm{AS}_{4}=0.4868, \\
& \mathrm{AS}_{5}=0.1109 .
\end{aligned}
$$

Step 8. According to the calculation results, the greater the value of $\mathrm{AS}_{i}$, the better the alternative, so the order of all alternatives is $P_{2}>P_{3}>P_{4}>P_{1}>P_{5} ; P_{2}$ is the best alternative.

\section{Conclusion}

In MAGDM methods, uncertainty and hesitation are usually unavoidable. PI2TLSs are more likely to reflect uncertain or ambiguous information. At the same time, the EDAS method has the characteristics of small computation and few steps. Therefore, this paper proposes the PI2TLN-EDAS method to solve the MAGDM problem of PI2TLNs. Then, taking ship navigation environment safety evaluation as an example, the effectiveness and practicability of the method are verified through comparative analysis. Further research will carry out other applications of this method in other uncertain MAGDM problems [65-68] and other fields [69-75].

\section{Data Availability}

The data used to support the findings of this study are included within the article.

\section{Conflicts of Interest}

The author declares that there are no conflicts of interest.

\section{References}

[1] M. K. Ghorabaee, E. K. Zavadskas, L. Olfat, and Z. Turskis, "Multi-criteria inventory classification using a new method of evaluation based on distance from average solution (EDAS)," Informatica, vol. 26, no. 3, pp. 435-451, 2015.

[2] L. Ustinovichius, E. K. Zavadskas, and V. Podvezko, "Application of a quantitative multiple criteria decision making (MCDM-1) approach to the analysis of investments in construction," Control and Cybernetics, vol. 36, pp. 251-268, 2007.

[3] V. Kersuliene, E. K. Zavadskas, and Z. Turskis, "Selection of rational dispute resolution method by applying new step-wise weight assessment ratio analysis (SWARA)," Journal of Business Economics and Management, vol. 11, pp. 243-258, 2010.

[4] E. K. Zavadskas, J. Antucheviciene, and P. Chatterjee, "Multiple-criteria decision-making (MCDM) techniques for business processes information management," Information, vol. 10, 2019.

[5] N. Liao, G. Wei, and X. Chen, "TODIM method based on cumulative prospect theory for multiple attributes group decision making under probabilistic hesitant fuzzy setting," International Journal of Fuzzy Systems, 2021.

[6] Z. Jiang, G. Wei, J. Wu, and X. Chen, "CPT-TODIM method for picture fuzzy multiple attribute group decision making and its application to food enterprise quality credit evaluation," Journal of Intelligent and Fuzzy Systems, vol. 40, no. 5, pp. 10115-10128, 2021.

[7] M. Zhao, G. Wei, C. Wei, and J. Wu, "Pythagorean fuzzy TODIM method based on the cumulative prospect theory for MAGDM and its application on risk assessment of science and technology projects," International Journal of Fuzzy Systems, vol. 23, no. 4, pp. 1027-1041, 2021.

[8] K. Devi, "Extension of VIKOR method in intuitionistic fuzzy environment for robot selection," Expert Systems with Applications, vol. 38, pp. 14163-14168, 2011.

[9] H. Liao and Z. Xu, "A VIKOR-based method for hesitant fuzzy multi-criteria decision making," Fuzzy Optimization and Decision Making, vol. 12, no. 4, pp. 373-392, 2013.

[10] H. Liao, G. Si, Z. Xu, and H. Fujita, "Hesitant fuzzy linguistic preference utility set and its application in selection of fire rescue plans," International Journal of Environmental Research and Public Health, vol. 15, 2018.

[11] S. H. Mirghafoori, M. R. Izadi, and A. Daei, "Analysis of the barriers affecting the quality of electronic services of libraries by VIKOR, FMEA and entropy combined approach in an intuitionistic-fuzzy environment," Journal of Intelligent and Fuzzy Systems, vol. 34, no. 4, pp. 2441-2451, 2018.

[12] D. Pamucar, I. Petrovic, and G. Cirovic, "Modification of the Best-Worst and MABAC methods: a novel approach based on interval-valued fuzzy-rough numbers," Expert Systems with Applications, vol. 91, pp. 89-106, 2018.

[13] Z.-p. Tian, J. Wang, J.-q. Wang, and H.-y. Zhang, "Simplified neutrosophic linguistic multi-criteria group decision-making approach to green product development," Group Decision and Negotiation, vol. 26, no. 3, pp. 597-627, 2017.

[14] Y. Yang and X. Peng, "A revised TOPSIS method based on interval fuzzy soft set models with incomplete weight information," Fundamenta Informaticae, vol. 152, no. 3, pp. 297-321, 2017.

[15] D. Liang, Z. Xu, D. Liu, and Y. Wu, "Method for three-way decisions using ideal TOPSIS solutions at Pythagorean fuzzy information," Information Sciences, vol. 435, pp. 282-295, 2018.

[16] X. Peng and J. Dai, "Approaches to single-valued neutrosophic MADM based on MABAC, TOPSIS and new similarity measure with score function," Neural Computing \& Applications, vol. 29, no. 10, pp. 939-954, 2018.

[17] G. Wang and Y. Duan, "TOPSIS approach for multi-attribute decision making problems based on n-intuitionistic polygonal 
fuzzy sets description," Computers \& Industrial Engineering, vol. 124, pp. 573-581, 2018.

[18] S. Zhang, H. Gao, G. Wei, and X. Chen, "Grey relational analysis method based on cumulative prospect theory for intuitionistic fuzzy multi-attribute group decision making," Journal of Intelligent and Fuzzy Systems, vol. 41, no. 2, pp. 3783-3795, 2021.

[19] E. K. Zavadskas, R. Bausys, and I. Mazonaviciute, "Safety evaluation methodology of urban public parks by multi-criteria decision making," Landscape and Urban Planning, vol. 189, pp. 372-381, 2019.

[20] D. Stanujkic, D. Karabasevic, E. K. Zavadskas, F. Smarandache, and F. Cavallaro, "An approach to determining customer satisfaction in traditional Serbian restaurants," Entrepreneurship and Sustainability Issues, vol. 6, no. 3, pp. 1127-1138, 2019.

[21] S. Meysam Mousavi, J. Antuchevičienè, E. K. Zavadskas, B. Vahdani, and H. Hashemi, "A new decision model for cross-docking center location in logistics networks under interval-valued intuitionistic fuzzy uncertainty," Transport, vol. 34, no. 1, pp. 30-40, 2019.

[22] C. Kahraman, M. Keshavarz Ghorabaee, E. K. Zavadskas, S. Cevik Onar, M. Yazdani, and B. Oztaysi, "Intuitionistic fuzzy edas method: an application to solid waste disposal site selection," Journal of Environmental Engineering and Landscape Management, vol. 25, no. 1, pp. 1-12, 2017.

[23] W.-Z. Liang, G.-Y. Zhao, and S.-Z. Luo, "An integrated EDAS-ELECTRE method with Picture fuzzy information for cleaner production evaluation in gold mines," IEEE Access, vol. 6, pp. 65747-65759, 2018.

[24] Z. Jiang, G. Wei, and X. Chen, "EDAS method based on cumulative prospect theory for multiple attribute group decision-making under picture fuzzy environment," Journal of Intelligent and Fuzzy Systems, vol. 47, pp. 1-13, 2021.

[25] X. Peng, J. Dai, and H. Yuan, "Interval-valued fuzzy soft decision making methods based on MABAC, similarity measure and EDAS," Fundamenta Informaticae, vol. 152, no. 4, pp. 373-396, 2017.

[26] Y. Huang, R. Lin, and X. Chen, "An enhancement EDAS method based on prospect theory," Technological and Economic Development of Economy, vol. 27, no. 5, pp. 1019-1038, 2021.

[27] G. Wei, C. Wei, and Y. Guo, "EDAS method for probabilistic linguistic multiple attribute group decision making and their application to green supplier selection," Soft Computing, vol. 25, no. 14, pp. 9045-9053, 2021.

[28] F. Lei, G. Wei, W. Shen, and Y. Guo, "PDHL-EDAS method for multiple attribute group decision making and its application to 3D printer selection," Technological and Economic Development of Economy, vol. 28, pp. 179-200, 2022.

[29] Z. Jiang, G. Wei, and Y. Guo, "Picture fuzzy MABAC method based on prospect theory for multiple attribute group decision making and its application to suppliers selection," Journal of Intelligent and Fuzzy Systems, vol. 42, pp. 1-11, 2022.

[30] D. Zhang, Y. Su, M. Zhao, and X. Chen, "CPT-TODIM method for interval neutrosophic MAGDM and its application to third-party logistics service providers selection," Technological and Economic Development of Economy, vol. 28, pp. 201-219, 2022.

[31] S. Wang, G. Wei, J. Lu, J. Wu, C. Wei, and X. Chen, “GRP and CRITIC method for probabilistic uncertain linguistic MAGDM and its application to site selection of hospital constructions," Soft Computing, vol. 26, no. 1, pp. 237-251, 2022.
[32] Y. Su, M. Zhao, G. Wei, C. Wei, and X. Chen, "Probabilistic uncertain linguistic EDAS method based on prospect theory for multiple attribute group decision-making and its application to green finance," International Journal of Fuzzy Systems, 2021.

[33] Y. Su, M. Zhao, C. Wei, and X. Chen, "PT-TODIM method for probabilistic linguistic MAGDM and application to industrial control system security supplier selection," International Journal of Fuzzy Systems, 2021.

[34] L. A. Zadeh, "Fuzzy sets," Information and Control, vol. 8, no. 3, pp. 338-353, 1965.

[35] K. T. Atanassov, "Intuitionistic fuzzy sets," Fuzzy Sets and Systems, vol. 20, no. 1, pp. 87-96, 1986.

[36] M. Zhao, G. Wei, X. Chen, and Y. Wei, "Intuitionistic fuzzy MABAC method based on cumulative prospect theory for multiple attribute group decision making," International Journal of Intelligent Systems, vol. 36, no. 11, pp. 6337-6359, 2021.

[37] L. Xiao, G. Wei, Y. Guo, and X. Chen, "Taxonomy method for multiple attribute group decision making based on intervalvalued intuitionistic fuzzy with entropy," Journal of Intelligent and Fuzzy Systems, vol. 41, no. 6, pp. 7031-7045, 2021.

[38] H. Garg, "Intuitionistic fuzzy hamacher aggregation operators with entropy weight and their applications to multi-criteria decision-making problems," Iranian Journal of Science and Technology, Transactions of Electrical Engineering, vol. 43, no. 3, pp. 597-613, 2019.

[39] H.-p. Chen and G.-q. Xu, "Group decision making with incomplete intuitionistic fuzzy preference relations based on additive consistency," Computers \& Industrial Engineering, vol. 135, pp. 560-567, 2019.

[40] J. Ye, "Generalized Dice measures for multiple attribute decision making under intuitionistic and interval-valued intuitionistic fuzzy environments," Neural Computing \& Applications, vol. 30, no. 12, pp. 3623-3632, 2018.

[41] S. Wan, F. Wang, and J. Dong, "A group decision-making method considering both the group consensus and multiplicative consistency of interval-valued intuitionistic fuzzy preference relations," Information Sciences, vol. 466, pp. 109-128, 2018.

[42] X. L. Tian, M. L. Niu, J. S. Ma, and Z. S. Xu, "A novel TODIM with probabilistic hesitant fuzzy information and its application in green supplier selection," Complexity, vol. 2020, Article ID 2540798, 2020.

[43] N. Liao, H. Gao, G. Wei, and X. Chen, "CPT-MABAC-Based multiple attribute group decision making method with probabilistic hesitant fuzzy information," Journal of Intelligent and Fuzzy Systems, vol. 41, no. 6, pp. 6999-7014, 2021.

[44] Y. Zhai, Z. Xu, and H. Liao, "Measures of probabilistic intervalvalued intuitionistic hesitant fuzzy sets and the application in reducing excessive medical examinations," IEEE Transactions on Fuzzy Systems, vol. 26, no. 3, pp. 1651-1670, 2018.

[45] F. Jiang and Q. Ma, "Multi-attribute group decision making under probabilistic hesitant fuzzy environment with application to evaluate the transformation efficiency," Applied Intelligence, vol. 48, no. 4, pp. 953-965, 2018.

[46] B. Farhadinia and Z. Xu, "Ordered weighted hesitant fuzzy information fusion-based approach to multiple attribute decision making with probabilistic linguistic term sets," Fundamenta Informaticae, vol. 159, no. 4, pp. 361-383, 2018.

[47] W. Zhou and Z. Xu, "Expected hesitant VaR for tail decision making under probabilistic hesitant fuzzy environment," Applied Soft Computing, vol. 60, pp. 297-311, 2017.

[48] X. Gou and Z. Xu, "Novel basic operational laws for linguistic terms, hesitant fuzzy linguistic term sets and 
probabilistic linguistic term sets," Information Sciences, vol. 372, pp. 407-427, 2016.

[49] G. Wei, R. Lin, J. Lu, J. Wu, and C. Wei, “The generalized dice similarity measures for probabilistic uncertain linguistic MAGDM and its application to location planning of electric vehicle charging stations," International Journal of Fuzzy Systems, 2021.

[50] N. Yue, D. Wu, J. Xie, and S. Chen, "Probabilistic linguistic multi-criteria decision-making based on double information under imperfect conditions," Fuzzy Optimization and Decision Making, vol. 19, no. 4, pp. 391-433, 2020.

[51] A. D. Shrivathsan, R. Krishankumar, A. R. Mishra, K. S. Ravichandran, S. Kar, and V. Badrinath, "An integrated decision approach with probabilistic linguistic information for test case prioritization," Mathematics, vol. 8, 2020.

[52] S. Ramadass, R. Krishankumar, K. S. Ravichandran, H. C. Liao, S. Kar, and E. Herrera-Viedma, "Evaluation of cloud vendors from probabilistic linguistic information with unknown/partial weight values," Applied Soft Computing, vol. $97,2020$.

[53] X. Mi, H. Liao, X. Wu, and Z. Xu, "Probabilistic linguistic information fusion: a survey on aggregation operators in terms of principles, definitions, classifications, applications, and challenges," International Journal of Intelligent Systems, vol. 35, no. 3, pp. 529-556, 2020.

[54] X. Gou, Z. Xu, H. Liao, and F. Herrera, "Probabilistic double hierarchy linguistic term set and its use in designing an improved VIKOR method: the application in smart healthcare," Journal of the Operational Research Society, vol. 72, no. 12, pp. 2611-2630, 2021.

[55] F. Lei, G. Wei, and X. Chen, "Model-based evaluation for online shopping platform with probabilistic double hierarchy linguistic CODAS method," International Journal of Intelligent Systems, vol. 36, no. 9, pp. 5339-5358, 2021.

[56] F. Lei, G. Wei, and X. Chen, "Some self-evaluation models of enterprise's credit based on some probabilistic double hierarchy linguistic aggregation operators," Journal of Intelligent and Fuzzy Systems, vol. 40, no. 6, pp. 11809-11828, 2021.

[57] R. R. Yager, "Pythagorean membership grades in multicriteria decision making," IEEE Transactions on Fuzzy Systems, vol. 22, no. 4, pp. 958-965, 2014.

[58] X. Zhang, "A novel approach based on similarity measure for pythagorean fuzzy multiple criteria group decision making," International Journal of Intelligent Systems, vol. 31, no. 6, pp. 593-611, 2016.

[59] S. Zeng, J. Chen, and X. Li, "A hybrid method for pythagorean fuzzy multiple-criteria decision making," International Journal of Information Technology and Decision Making, vol. 15 , no. 02 , pp. $403-422,2016$.

[60] H. Garg, "Confidence levels based Pythagorean fuzzy aggregation operators and its application to decision-making process," Computational \& Mathematical Organization Theory, vol. 23, no. 4, pp. 546-571, 2017.

[61] Y.-H. Huang and G.-W. Wei, "TODIM method for Pythagorean 2-tuple linguistic multiple attribute decision making," Journal of Intelligent and Fuzzy Systems, vol. 35, no. 1, pp. 901-915, 2018.

[62] R. R. Yager and A. M. Abbasov, "Pythagorean membership grades, complex numbers, and decision making," International Journal of Intelligent Systems, vol. 28, no. 5, pp. 436$452,2013$.

[63] F. Herrera and L. Martinez, "A 2-tuple fuzzy linguistic representation model for computing with words," IEEE Transactions on Fuzzy Systems, vol. 8, pp. 746-752, 2000.
[64] T. T. He, G. W. Wei, R. Lin, J. P. Lu, C. Wei, and J. Wu, "Pythagorean interval 2-tuple linguistic VIKOR method for evaluating human factors in construction project management," Iranian Journal of Fuzzy Systems, vol. 17, pp. 93-105, 2020.

[65] P. H. Thong and L. H. Son, "Picture fuzzy clustering: a new computational intelligence method," Soft Computing, vol. 20, no. 9, pp. 3549-3562, 2016.

[66] L. H. Son, "Measuring analogousness in picture fuzzy sets: from picture distance measures to picture association measures," Fuzzy Optimization and Decision Making, vol. 16, no. 3, pp. 359-378, 2017.

[67] E. K. Zavadskas, J. Antucheviciene, J. Saparauskas, and Z. Turskis, "MCDM methods WASPAS and MULTIMOORA: verification of robustness of methods when assessing alternative solutions," Economic Computation \& Economic Cybernetics Studies \& Research, vol. 47, pp. 5-20, 2013.

[68] M. Zhao, G. Wei, Y. Guo, and X. Chen, "CPT-TODIM method for interval-valued bipolar fuzzy multiple attribute group decision making and application to industrial control security service provider selection," Technological and Economic Development of Economy, vol. 27, no. 5, pp. 1186-1206, 2021.

[69] R. M. Rodríguez, H. Liu, and L. Martínez, “A fuzzy representation for the semantics of hesitant fuzzy linguistic term sets," in Foundations of Intelligent Systems, Z. Wen and T. Li, Eds., pp. 745-757, 2014.

[70] F. J. Estrella, R. M. Rodriguez, and L. Martinez, "A hesitant linguistic fuzzy TOPSIS approach integrated into FLINTSTONES," in Proceedings of the 2015 Conference of the International Fuzzy Systems Association and the European Society for Fuzzy Logic and Technology, J. M. Alonso, H. Bustince, and M. Reformat, Eds., pp. 799-806, 2015.

[71] E. K. Zavadskas, A. Kaklauskas, and T. Vilutiené, "Multicriteria evaluation of apartment blocks maintenance contractors: Lithuanian case study," International Journal of Strategic Property Management, vol. 13, no. 4, pp. 319-338, 2009.

[72] R. A. Krohling, A. G. C. Pacheco, and G. A. dos Santos, "TODIM and TOPSIS with Z-numbers," Frontiers of Information Technology \& Electronic Engineering, vol. 20, no. 2, pp. 283-291, 2019.

[73] K.-w. Shen and J.-q. Wang, "Z-VIKOR method based on a new comprehensive weighted distance measure of Z-number and its application," IEEE Transactions on Fuzzy Systems, vol. 26, no. 6, pp. 3232-3245, 2018.

[74] S. Ezadi and T. Allahviranloo, "New multi-layer method for Z-number ranking using hyperbolic tangent function and convex combination," Intelligent Automation and Soft Computing, vol. 24, no. 1, pp. 217-221, 2018.

[75] R. A. Aliev, W. Pedrycz, and O. H. Huseynov, "Functions defined on a set of Z-numbers," Information Sciences, vol. 423, pp. 353-375, 2018. 